\title{
Evaluation of Lipid-Based Carrier Systems and Inclusion Complexes of Diethyldithiocarbamate-Iron to Trap Nitric Oxide in Biological Systems
}

\author{
Nicolas Charlier, ${ }^{1}$ Véronique Préat,${ }^{2}$ and Bernard Gallez ${ }^{1 *}$
}

\begin{abstract}
The success of spin trapping techniques in vivo hinges on whether spin traps with high trapping efficiency and biocompatibility can be developed. Currently, two iron chelates based on the dithiocarbamate structure (hydrophilic ferro-di( $N$-methyl-D-glucamine-dithiocarbamate, or Fe(II)-MGD, and lipophilic ferro-di(diethyldithiocarbamate), or Fe(II)-DETC), are used for spin trapping of nitric oxide (NO) in biologic systems. However, detection efficiency is hampered by a complex redox chemistry for Fe(II)-MGD and by the insolubility of Fe(II)-DETC in water. To circumvent these problems, two new spin trap formulations based on Fe(II)-DETC were developed: a lipid-based carrier system stabilized by lecithin and inclusion complexes in hydroxypropyl- $\beta$-cyclodextrin. The capability of these two systems to trap NO was determined and compared to the standard spin traps in vitro (in the presence of an NO donor) and in vivo (after induction of septic shock in mice). The sensitivity of the detection of NO was significantly increased (by a factor of 4) using the lipid-based carrier systems or inclusion complexes compared to the standard spin trap agents. Magn Reson Med 55:215-218, 2006. (c) 2005 Wiley-Liss, Inc.
\end{abstract}

Key words: EPR; ESR; nitric oxide; spin trapping; in vivo

Nitric oxide (NO) is a key messenger and regulator in many physiologic pathways as well as in pathologic states (1). The role of nitric oxide in tumor biology is complex, and the biologic response depends on where, when, and how much NO is produced (2). The radiosensitizing properties of nitric oxide have also recently been investigated $(3,4)$. Because of this essential role in biology and therapeutics, several methods for quantification of NO in biologic media have been developed (5).

Among these techniques, electron paramagnetic resonance (EPR) spin trapping is very appealing because it offers very high sensitivity, high selectivity, and the possibility for application in vivo (6). To be detectable in biologic media, NO should react with a spin trapping agent, forming a free radical adduct with a longer stability. The most commonly used spin traps for the detection of

${ }^{1}$ Laboratory of Medicinal Chemistry and Radiopharmacy, Biomedical Resonance Unit, Université Catholique de Louvain, Brussels, Belgium.

${ }^{2}$ Laboratory of Pharmaceutical Technology, Université Catholique de Louvain, Brussels, Belgium.

Grant sponsor: Belgian National Fund for Scientific Research (F.N.R.S.); Gran sponsor: the F.R.S.M.; Grant sponsor: the F.R.I.A; Grant sponsor: the Fonds Joseph Maisin; Grant sponsor: the "Actions de Recherches ConcertéesCommunauté Française de Belgique; Grant number: ARC 04/09-317; Grant sponsor: NIH; Grant numbers: P01 EB002180 and P41 EB002032.

${ }^{*}$ Correspondence to: Bernard Gallez, CMFA/REMA, Avenue E. Mounier, 73.40, B-1200 Brussels, Belgium. E-mail: gallez@cmfa.ucl.ac.be

Received 15 July 2005; revised 12 September 2005; accepted 14 September 2005.

DOI $10.1002 / \mathrm{mrm} .20746$

Published online 8 December 2005 in Wiley InterScience (www.interscience. wiley.com).

(c) 2005 Wiley-Liss, Inc.
NO are iron(II) chelates for which the ligand possess a dithiocarbamate structure (7). Two examples are the hydrophilic compound $N$-methyl-D-glucamine diethyldithiocarbamate (MGD) (8) and the lipophilic compound diethyldithiocarbamate (DETC) (9). Fe(II)-DETC is the most commonly used spin trap in cell cultures. Although Fe(II)DETC is insoluble in water, it is able to trap NO and relocate in cell membranes without precipitation (10). For in vivo studies, however, Fe(II)-DETC cannot be administered by i.v. (IV) injection because the aggregates formed in the blood stream cause embolisms. To get around this problem, attempts have been made to inject the iron and DETC components separately into the animals using two different sites of injection: DETC via the intraperitoneal (i.p.) route and iron via the subcutaneous (s.c.) route (11). However, this strategy led to problems of reproducibility in the quantification of NO in tissues, since the quantification depends on the absorption of each individual compound and on the kinetics of formation of the complex in vivo. Theoretically, the Fe(II)-MGD complex is more suitable than Fe(II)-DETC for in vivo investigations because it is freely soluble in aqueous environments and can be administered by IV without problems. However, its in vivo trapping efficiency has been reported to be poor because of a tendency of the $\mathrm{NO}$ adduct to precipitate and therefore lose EPR signal (12). Moreover, it has also been demonstrated that Fe(II)-MGD undergoes a very complex redox chemistry, leading to difficulties in the assessment of the local production of NO (13).

To overcome these problems, we developed two novel formulations of the Fe(II)-DETC complex that can be administered by IV injection. These formulations are based on technologies commonly used in the pharmaceutical industry for the solubilization of hydrophobic compounds (14). The first method is a lipid-based carrier system stabilized with lecithin $(15,16)$. The second is based on the formation of inclusion complex using hydroxypropyl- $\beta$ cyclodextrin (17). Cyclodextrins are bucket-shape oligosaccharides composed of glucose units (seven units for $\beta$-cyclodextrin). The conformation of the glucose units in the cyclodextrin arranges the hydrophilic hydroxyl groups outward toward the external environment and most of the hydrophobic groups inward toward the inside of the cavity. This unique configuration enables the molecule to form inclusion complexes with lipophilic compounds, with many applications in pharmaceutical technology for solubilizing drugs (18). Here, we describe the methods of formulation of these new complexes. Furthermore, we present data on the capability of these new complexes to trap NO in vitro and in vivo compared to standard Fe(II)DETC and Fe(II)-MGD. 


\section{METHODS}

\section{Spin Trap Formulations}

MGD sodium salt and (2-( $N, N$-Diethylamino)-diazenolate2-oxide diethylammonium salt (DEA NONOate) were purchased from Alexis Benelux (Zandhoven, Belgium). All other reagents were purchased from Sigma-Aldrich (Bornem, Belgium). All procedures were carried out under nitrogen to prevent iron(II) from oxidizing. The solvents were degassed with nitrogen before use. In all preparations, we used an excess of ligand with respect to iron (ligand:Fe(II) ratio $=5: 1$ ).

\section{Lipid-Based Carrier System}

A total of $360 \mathrm{mg}$ of L- $\alpha$-phosphatidylcholine from egg yolk was first dissolved in $10 \mathrm{~mL}$ chloroform/methanol (2/1) in a 500-mL round-bottom flask. The solvent was then evaporated under vacuum with a rotavapor for $45 \mathrm{~min}$ to form a lipid film. The film was hydrated with $2.25 \mathrm{~mL}$ water in the round-bottom flask for $45 \mathrm{~min}$. Next, $1.5 \mathrm{~mL}$ was collected and sonicated with a VibraCell 100 (Sonics \& Materials, Danbury, CT, USA) for $1.5 \mathrm{~min}$ at an output power of $20 \mathrm{~W}$. Two independently prepared solutions of 0.25 $\mathrm{mL}$ DETC sodium salt (500 mM in water) and of $0.25 \mathrm{~mL}$ iron(II) sulfate (100 $\mathrm{mM}$ in water) were then added to the hydrated lipids and vigorously stirred. The lipid-based carrier system was filtered with a $0.45-\mu \mathrm{m}$ filter. The particle size was checked by photon correlation spectroscopy using a Zetasizer NanoSeries (Malvern Intruments, Malvern, United Kingdom).

\section{Inclusion Complex}

A total of $0.25 \mathrm{~mL}$ of DETC sodium salt (250 mM in water) and $0.25 \mathrm{~mL}$ of iron(II) sulfate $(50 \mathrm{mM}$ in water) were added under vigorous stirring to $0.5 \mathrm{~mL}$ of a hydroxypropyl- $\beta$-cyclodextrin solution $(60 \% \mathrm{w} / \mathrm{v}$ in water).

\section{Preparation of Other Spin Trap Solutions}

The lipid-based carrier system and inclusion complex were compared with the standard Fe(II)-MGD and Fe(II)DETC spin traps in vitro and in vivo. Fe(II)-MGD complex was prepared by dissolution in water of MGD sodium salt (62.5 mM) and iron(II) sulfate (12.5 mM). Because Fe(II)DETC is insoluble in water, we prepared two distinct water solutions: The first solution was DETC (250 mM). The second solution was iron(II) sulfate (50 mM) together with sodium citrate dihydrate (250 mM) (11).

\section{In Vitro Spin Trapping}

This experiment was performed to assess the capability of the different systems to trap NO in an aqueous environment. The spin trap solution was added to DEA NONOate, an NO donor. The final concentrations were 10, 12.5, and $62.5 \mathrm{mM}$ for DEA NONOate, Fe(II), and ligand, respectively. The mixture was stirred for $1 \mathrm{~min}$, and the sample was put in a capillary tube that was sealed at both ends. The measurements were done on a Bruker EMX-EPR spectrometer (operating at $9 \mathrm{GHz}) 30$ and 60 min after mixing. The acquisitions were performed at $310 \mathrm{~K}$. The spectrom- eter settings were field sweep $60 \mathrm{Mt}$; power $4 \mathrm{~mW}$; modulation amplitude $0.15 \mathrm{mT}$; scan time $20 \mathrm{~s}$; number of scans 20. The signal intensity was estimated by measuring the area under the curve of the spectra by double integration using the Bruker WinEPR program.

\section{In Vivo Experiments}

To assess the capability of the formulations to trap NO in vivo, we used a septic shock model in mice which induces a large release of NO (19). Lipopolysaccharides (LPS) (from Escherichia coli, serotype 055:B5) were administered by IP route $(50 \mathrm{mg} / \mathrm{kg}$ ) to male $\sim 25 \mathrm{~g}$ NMRI mice (Animalerie facultaire, Faculté de Médecine, Catholic University of Louvain). Six hours later, the spin traps were administered: $0.25 \mathrm{mmol} / \mathrm{kg}$ of Fe(II), $1.25 \mathrm{mmol} / \mathrm{kg}$ of ligand. The Fe(II)-MGD complex, the lipid-based carrier system, and the inclusion complex containing Fe(II)-DETC were administered by i.v. route. The "classic" double injection protocol was also used, with DETC injected via the i.p. route and Fe(II) injected via the s.c. route. The mice were sacrificed at different times after the administration of spin traps $(5,10,15,20,30,45,60,120 \mathrm{~min})$. The blood was collected through the cut, open end of a translucent Tiemann catheter (Maersk Medical Ltd.) having an inner diameter of $4.35 \mathrm{~mm}$. The catheter was filled to a height of $6.5 \mathrm{~cm}$ and clamped at its end before being placed gently into liquid nitrogen. The length of the cylindrical sample was longer than the active region of the cavity, so that the filling factor for all samples was essentially identical. The catheter was emptied by removing the "carrot" of frozen blood in a liquid nitrogen cooled dewar. Samples located in the lower part of the dewar were separated from the liquid nitrogen in the upper part of the dewar by a wad of cotton and then analyzed using a Magnettech Miniscope spectrometer MS-200 operating at $9 \mathrm{GHz}$. The following settings were used for this spectrometer: field sweep 10 $\mathrm{mT}$; power $10 \mathrm{~mW}$; modulation amplitude $0.15 \mathrm{mT}$; scan time $20 \mathrm{~s}$; number of scans 10. The signal-to-noise ratio of the spectra was measured. The liver and the kidneys were also sampled 30 min after the injection of the spin traps (time of maximal values recorded in the blood). Mice were sacrificed, and the liver and kidneys were excised, weighed, mixed with $\mathrm{NaCl} 0.9 \%$ ( $1 \mathrm{~mL} / \mathrm{g}$ of tissue), and homogenized with a Potter-Elvehjem tissue grinder. The homogenate was collected by a Tiemann catheter and frozen in liquid nitrogen as previously described. The total time for organ extraction and freezing was approximately 5 min. The measurements were performed as previously described on a Bruker EMX-EPR spectrometer using the following settings: field sweep $10 \mathrm{mT}$; power $40 \mathrm{~mW}$; single scan of $20 \mathrm{~s}$.

\section{RESULTS AND DISCUSSION}

We report here rapid and convenient methods for preparing injectable forms of Fe(II)-DETC. We developed a lipidbased carrier system containing Fe(II)-DETC stabilized by lecithin, a surfactant commonly used in emulsions for parenteral administration. Using this procedure, we obtained dark, brown, milky colloids of Fe(II)-DETC. We reproducibly obtained particles with a size of $270 \pm 10$ 


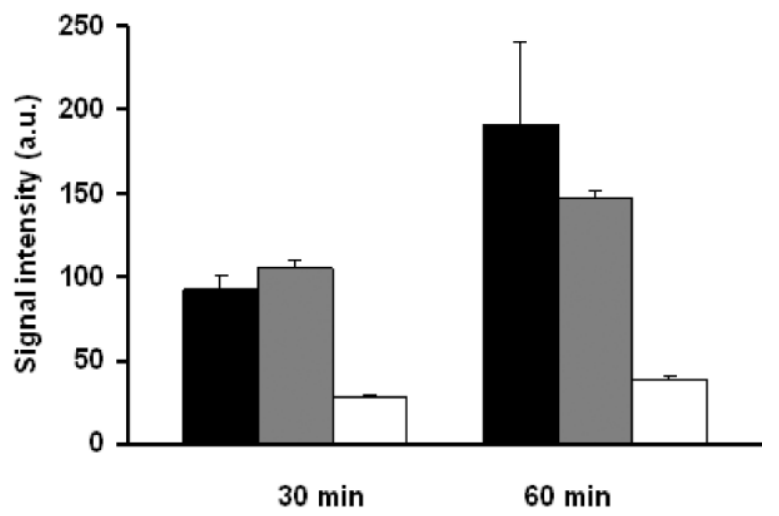

FIG. 1. Ability of the iron complexes to trap nitric oxide in vitro: signal intensity, corresponding to the area under the curve of EPR spectra (mean \pm SEM, $n=3$ ), after mixing the NO donor DEA NONOate with Fe(II)-DETC lecithin (black column), Fe(II)-DETC hydroxypropyl- $\beta$-cyclodextrin (gray column), and Fe(II)-MGD (white column). Left: signal intensity $30 \mathrm{~min}$ after mixing. Right: signal intensity after $60 \mathrm{~min}$.

$\mathrm{nm}$. We also used cyclodextrins, which allow inclusion of hydrophobic molecules in the center of the molecule. Based on the hydroxypropyl- $\beta$-cyclodextrin molecular structure, the typical molecular size for these inclusion complexes is less than $2 \mathrm{~nm}$. Using this procedure, we obtained dark-brown translucent solutions of Fe(II)-DETC.

The capability of the different systems to trap NO in an aqueous environment was evaluated by incubation in the presence of DEA NONOate as an NO donor. The results are presented in Fig. 1. Using both systems, the EPR signal intensity was about 4 times higher compared to the hydrophilic complex Fe(II)-MGD (significant difference, $t$ test, $P<0.05$ ). It should be emphasized that without lecithin or hydroxypropyl- $\beta$-cyclodextrin, the mixing of solutions of DETC and Fe(II) led immediately to precipitation and loss of EPR signal. The inferior signal of MGD-Fe(II)-NO was likely due to its rapid degradation and complex redox chemistry, as previously described by others $(12,13)$.

The ability of our novel complexes to trap NO in vivo was evaluated in a model of sepsis. Comparisons were made with $\mathrm{Fe}$ (II)-MGD and the double injection protocol (DETC injected via the i.p. route and Fe(II) via the s.c. route) (19). In Fig. 2, we present the kinetics of the NO signal in the blood. The evolution of the concentration of NO adduct in the blood was variable, depending on the spin trap used in the experiment. The inclusion complex of $\mathrm{Fe}(\mathrm{II})$-DETC in cyclodextrin was able to trap NO very quickly. The signal intensity was already elevated only $5 \mathrm{~min}$ after injection and remained stable for up to $30 \mathrm{~min}$ before eventually decreasing. Using the lipid-based carrier system, the NO adduct was more slowly formed in the blood, with a plateau reached after about $15 \mathrm{~min}$. The concentration of $\mathrm{NO}$ adduct in the blood remained elevated for a longer time (up to $60 \mathrm{~min}$ ) before declining. This kinetics pattern is likely the result of the progressive trapping of NO during circulation in the blood stream, followed by removal of the adduct by metabolism and/or elimination. Because NO is more rapidly trapped by the inclusion complex than by the lipid based carrier system, we hypothesize that the iron center is more easily accessible to bind NO in the inclusion complex. The more rapid decay of the NO adduct for the inclusion complex could result from a more rapid metabolism (also linked to an easier accessibility to the radical center) or a more rapid elimination of the cyclodextrin from the blood stream. Interestingly, both formulations provide a significantly higher NO trapping efficiency than either MGD-Fe(II) or the double injection protocol of Fe(II)-DETC (two-way ANOVA).

We also performed spin trapping experiments in the liver and kidneys at the time of the maximal level of NO detected (30 min after the injection of the spin traps). The results are shown in Fig. 3. Typical EPR spectra obtained in $20 \mathrm{~s}$ are presented at two magnifications. Using a large sweep width (Fig. 3a), different components can be observed. Among them we can distinguish the signal from the Cu-DETC complex (20). The part of the spectrum corresponding to the NO-adduct is enlarged in Fig. 3b. The ranking in the efficiency for trapping NO in the liver was as follows: lipid-based carrier of Fe(II)-DETC $>$ double injection of Fe(II)-DETC > Fe(II)-MGD > inclusion complex of Fe(II)-DETC. The high level of NO detected in the liver by the lipid-based carrier system is consistent with the fact that these particles have a size that makes them easily phagocytosed by the reticuloendothelial system (Kuppfer cells in the liver). The amount of NO found in the kidney was lower than in the liver using all spin traps. Again, the lipid-based carrier system was the most efficient NO trap in this organ.

\section{CONCLUSION}

The success of spin trapping techniques in vivo hinges on the development of spin traps that are well characterized, with high trapping efficiency and biocompatibility. In this study, we provide two new formulations of Fe(II)-DETC that could be used for parenteral injections: inclusion complexes and a lipid-based carrier system. The prepara-

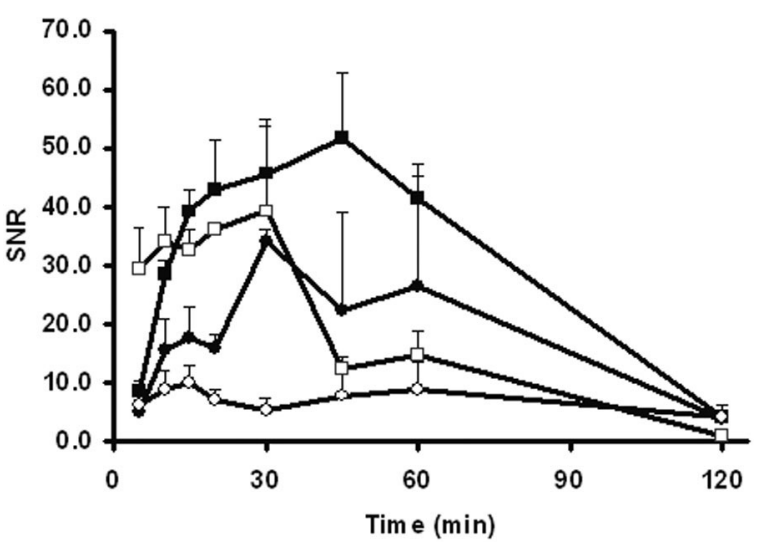

FIG. 2. Evolution of the EPR signal in the blood of mice treated with LPS after i.v. injection of different spin traps. The signal-to-noise ratio (mean \pm SEM, $n=3$ ) was measured from blood samples frozen in liquid nitrogen after i.v. injection of spin traps: Fe(II)-DETC lecithin ( $\square$ ), Fe(II)-DETC hydroxypropyl- $\beta$-cyclodextrin ( $\square$ ), Fe(II)DETC citrate (๑), and Fe(II)-MGD (O). 

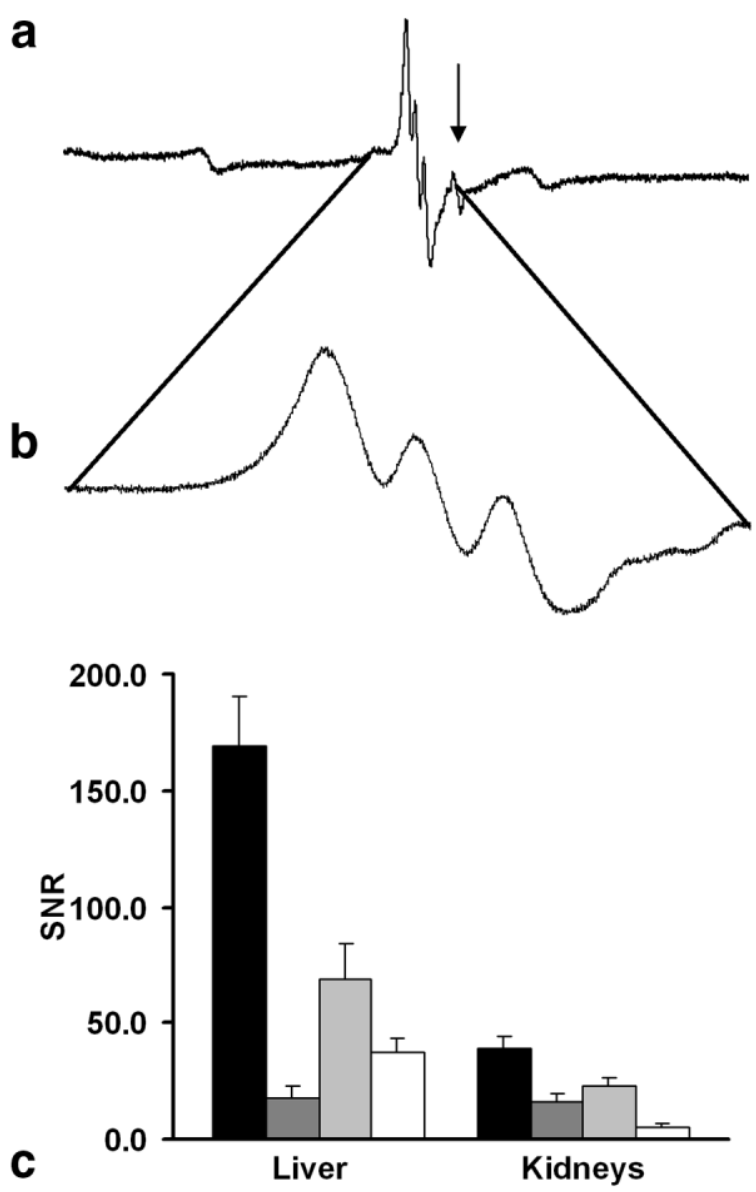

FIG. 3. Spin trapping in liver and kidneys of mice treated with LPS after injection of different spin traps. (a) Typical EPR spectrum from a liver homogenate in liquid nitrogen. The liver was sampled $30 \mathrm{~min}$ after IV administration of Fe(II)-DETC lecithin. The arrow indicates the part of the signal assigned to the Cu-DETC complex. (b) Part of the EPR spectrum presented in (a) that corresponds to the signal of the DETC-Fe(II)-NO adduct. (c) EPR signal intensity (signal-to-noise ratio, mean $\pm \mathrm{SEM}, n=6$ ) recorded in frozen liver homogenates (left columns) and in kidney homogenates (right columns) 30 min after i.v. injection of spin traps: Fe(II)-DETC lecithin (black columns), $\mathrm{Fe}(\mathrm{II})$-DETC hydroxypropyl- $\beta$-cyclodextrin (dark gray columns), $\mathrm{Fe}$ (II)-DETC citrate (light gray columns), and Fe(II)-MGD (white columns)

tion methods are simple and reproducible. The NO trapping efficiency in aqueous medium was 4 times higher for the new formulations compared to the standard spin trap Fe(II)-MGD. In vivo, the inclusion complex of Fe(II)-DETC was able to trap NO rapidly and continuously for at least 30 min after injection. The formation of NO adducts using the lipid-based carrier system was more gradual in the blood. After $30 \mathrm{~min}$, the lipid-based system provides the highest NO trapping efficiency in the liver and kidneys. The significant improvement in the yield of NO trapping should be beneficial for any research involving physiologic or pathophysiological phenomena associated with subtle changes in the level of nitric oxide.

\section{REFERENCES}

1. Blaise GA, Gauvin D, Gangal M, Authier S. Nitric oxide, cell signaling and cell death. Toxicology 2005;208:177-192.

2. Winck DA, Vodovotz Y, Laval J, Laval F, Dewhirst M, Mitchell JB. The multifaceted roles of nitric oxide in cancer. Carcinogenesis 1998;19: 711-721.

3. Jordan BF, Sonveaux P, Feron O, Grégoire V, Beghein N, Dessy C, Gallez B. Nitric oxide as a radiosensitizer: evidence for an intrinsic role in addition to its effect on oxygen delivery and consumption. Int J Cancer 2004;109:768-773.

4. Mitchell JB, DeGraaf W, Kim S, Cook JA, Gamson J, Christodoulou D, Feelish M, Vinck DA. Radiation sensitization by nitric oxide releasing agents. Br J Cancer 1996;27:S181-S184.

5. Tarpey MM, Wink DA, Grisham MB. Methods for detection of reactive metabolites of oxygen and nitrogen: in vitro and in vivo considerations. Am J Physiol Regul Integr Comp Physiol 2004;286:431-444.

6. Fujii H, Berliner LJ. Detection of bioradicals by in vivo L-Band electron spin resonance spectrometry. NMR Biomed 2004;17:311-318.

7. Yoshimura T, Yokoyama H, Fujii S, Takayama F, Oikawa K, Kamada H. In vivo EPR detection and imaging of endogenous nitric oxide in lipopolysaccharide-treated mice. Nat Biotechnol 1996;14:992-994.

8. Lai CS, Komarov AM. Spin trapping of nitric oxide produced in vivo in septic-shock mice. FEBS Lett 1994;345:120-124.

9. Tsuchiya K, Takasugi M, Minakuchi K, Fukuzawa K. Sensitive quantitation of nitric oxide by EPR spectroscopy. Free Rad. Biol Med 1996; 21:733-737.

10. Kleschyov AL, Mollnau H, Oelze M, Meinertz T, Huang Y, Harrison DG, Munzel T. Spin trapping of vascular nitric oxide using colloid Fe(II)-Diethyldithiocarbamate. Biochem Biophys Res Commun 2000 275:672-677.

11. Fujii H, Berliner JL. Ex vivo detection of nitric oxide in brain tissue. Mag Reson Med 1999;42:599-602.

12. Pou S, Tsai P, Porasuphatana S, Halpern HJ, Chandramouli GVR, Barth ED, Rosen GM. Spin trapping of nitric oxide by ferro-chelates: kinetic and in vivo pharmacokinetic studies. Biochim Biophys Acta 1999; 1427:216-226

13. Vanin AF, Liu X, Samouilov A, Stukan RA, Zweier JL. Redox properties of iron-dithiocarbamates and their nitrosyl derivatives: implications for their use as traps of nitric oxide in biological systems. Biochim Biophys Acta 2000;1474:365-77.

14. Strickley GR. Solubilizing excipient in oral and injectable formulations. Pharm Res 2004;21:201-230.

15. Gregoriadis G. The carrier potential of liposomes in biology and medecine (first of two parts). N Engl J Med 1976;295:704-710.

16. Gregoriadis G. The carrier potential of liposomes in biology and medecine (second of two parts). N Engl J Med 1976;295:765-770.

17. Freundenberg K, Cramer F, Plieninger H. Inclusion compounds of physiologically active organic compounds. 1953. German Pat. 895,769.

18. Loftsson T, Brewster EM. Pharmacieutical Applications of Cyclodextrins. 1. Drug solubilization and stabilization. J Pharm Sci 1996;85: 1017-1025.

19. James PE, Thomas MP, Jackson SK. Tissue oxygenation in sepsis; new insights from in vivo EPR. NMR Biomed 2004;17:319-326.

20. Komarov AM, Lai C-S. Detection of nitric oxide production in mice by spin-trapping electron paramagnetic resonance spectroscopy. Biochim Biophys Acta 1995;1272:29-36. 